\title{
A semi-empirical procedure for the evaluation of multi-stage turbine performances
}

\author{
D. Barsi, R. Canepa and A. Satta \\ Dipartimento di Macchine Sistemi Energetici e Trasporti \\ DIMSET, University of Genoa \\ Via Montallegro 1, 16145 Genoa (Italy) \\ Phone number: +0039 010353 2563, Fax number: +0039010353 2566, e-mail: dario.barsi@unige.it, ansat@unige.it
}

\begin{abstract}
The available tools for the analysis of fluiddynamic processes inside turbomachines are nowadays extremely powerful and versatile. Despite this, the use of simplified procedures, such as the $2 \mathrm{D}$ meridional approach, is still relevant, due to its great running simplicity and good accuracy. The full three-dimensional analysis, in fact, even if it could model thoroughly details the fluid-dynamic phenomena occurring in turbomachines, requires huge computational resources and longer computational times with respect to two-dimensional approaches, and this is justified only after a simplified preliminary study of the machine. Also management simplicity, consistence of the required data and usability of results are aspects that should not be ignored, above all for the possibility of creating tools easily available for turbomachines designers. A through flow method, formulated in terms of pressure rather than velocity, for the analysis of multistage axial turbines is presented. It is designed to provide reliable radial distributions of circumferentially averaged values for the fundamental unknowns. The proposed form of the radial equilibrium equation can represent also sharp velocity gradients in radial direction and deal with very realistic situations as discussed in the applications presented. The code has been used for the analysis of both an experimental multistage gas turbine and an industrial steam turbine. The results obtained have been compared with the circumferentially averaged results of a viscous fully $3 \mathrm{D}$ commercial code.
\end{abstract}

\section{Key words}

Through-flow, Turbine, Correlations, Combined Cycle, Semi-empirical.

\section{Introduction}

The aim of this work is to realize a semi-empirical method able to predict the performances of a small steam turbine that can be employed in combined cycle power plants for ship propulsion. In fact, this kind of plants are put forward in order to improve the overall efficiency and thus reduce emissions. Throughflow calculations are widely employed in industrial applications due to the high stability of the procedure and to its accuracy in terms of results [1].

Moreover it is important that these procedures present high stability, robustness and quick convergence rate in order to create easily available tools for preliminary analysis of turbomachines.

These procedures are in most cases semi-empirical, since they employ experimental correlations both for losses and for row exit angle evaluation. Losses and angles are usually taken as distributed in a uniform way in span wise direction. These methods provide overall results in accordance with those obtained from 3D CFD calculations, but this agreement cannot be always obtained also for the local values of fundamental unknowns.

Several throughflow procedures which employ local (and not overall) correlations both for losses and flow angle evaluation have been proposed in the open literature. Despite this, in order to evaluate the spanwise distributions of thermodynamic unknowns, it is fundamental that the method has high stability and robustness in order to properly evaluate high spanwise gradients caused by secondary effects, which in turn provide high variations of basic unknowns close to both hub and shroud.

\section{Calculation model}

For the resolution of the analysis problem it is assumed that the whole geometrical parameters are given. In the general formulation, in order to get the flow conditions in each point of the machine, it is necessary to calculate two thermodynamic variables and the three components of the velocity vector. Thus, if no further simplifying hypothesis reduces the problem dimension, the fundamental unknowns are five, and so the fundamental equations, which enable to solve the problem, have to be five. The calculation model is based upon the fundamental assumption of steady flow, axisymmetric flow with axisymmetric flow surfaces and adiabatic 
process [2]. Besides an axisymmetric cylindrical coordinate system $(\theta, z, R)$, sketched in Fig. 1, has been adopted.

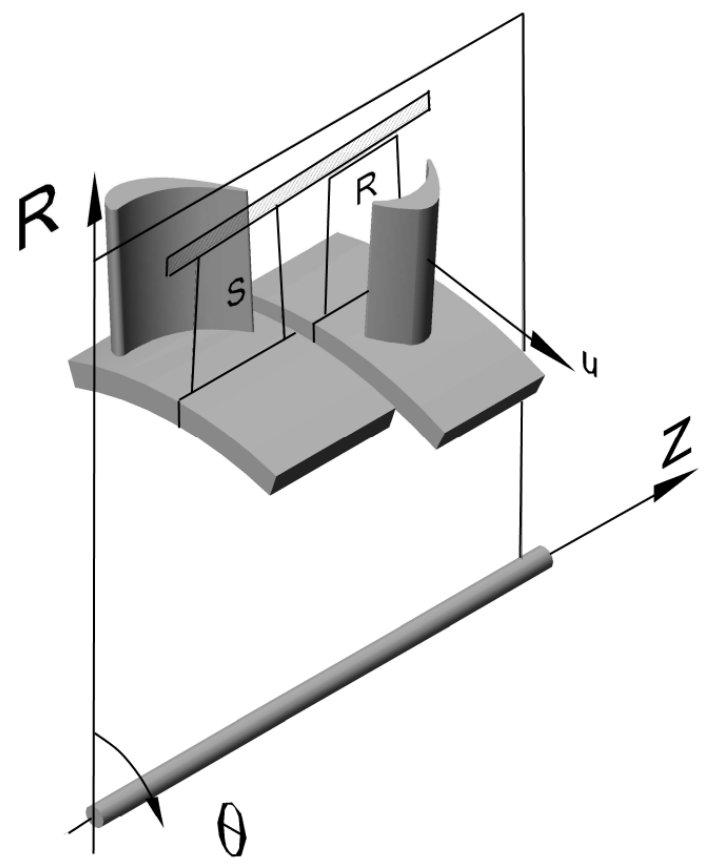

Fig.1. Coordinate system $(\theta, z, R)$ for machine representation

The streamline slope is assumed constant or with small variations in each control station, and its value can be estimated from the geometrical characteristics of the machine which are known in the analysis approach. Moreover we choose to study the stator rows in the absolute reference frame, while the rotor rows are investigated in the relative reference frame. This assumption leads to employ the same equations set for both type of rows, but on the other hand it requires the introduction of equations, that we call "changing equations" which allow to transfer the fundamental equation from a reference frame to the other. In addition two control parameters, which denote the operating conditions of the machine, have to be chosen: the rotational speed $n$ and the outlet static pressure $p_{u}$. Also inlet boundary conditions have to be assigned and the total quantities are assumed. A further simplifying assumption is considered: the calculation stations are set outside the blade channel. As a result of all the listed assumptions the problem dimensions change from three (general case) to two, and the fundamental unknowns from five to four. So one has to choose only four fundamental equations instead of five. Moreover if additional unknowns, called auxiliary unknowns, will be introduced into the fundamental equations, it will be necessary to introduce as many equations as the number of auxiliary unknowns.

\section{A. Fundamental and auxiliary equations}

It is convenient [2] to assume as fundamental unknowns the total enthalpy $H$, the total pressure $P$, the velocity $v$ and the flow angle $\varepsilon$ in the absolute reference frame for stator rows and in the relative reference frame for rotor rows. Thus, the fundamental equations, chosen for the problem posing, are the continuity equation in integral form, the energy equation in thermodynamic form and two correlation equations providing the flow angle and the total to total energy loss, which depends on the total pressure drop through the row.

If we call "reference frame unknowns" the four absolute unknowns for the case of the stator rows or the relative unknowns in the case of the rotor rows, the choice to study the rotor in the relative reference frame allows to use a general set of equations for both kind of rows (stator or rotor). So the employed equations are:

$$
\begin{gathered}
H_{2}\left(r_{2}\right)=H_{1}\left(r_{1}\right) \\
\int_{R_{i}}^{R_{e}} v_{2}\left(r_{2}\right) \rho_{2}\left(r_{2}\right) \cos \varepsilon_{2}\left(r_{2}\right) r d r=\dot{m}_{2}=\dot{m}_{1}-\dot{m}_{f}=\dot{m}_{1}(1-\delta) \\
P_{2}\left(r_{2}\right)=P_{1}\left(r_{1}\right) \sigma_{1,2}=P_{1}\left(r_{1}\right) f_{\sigma}\left(t_{f i}, \bar{t}_{g j}\right) \\
\varepsilon_{2}\left(r_{2}\right)=f_{\varepsilon}\left(t_{f l}^{\prime}, \bar{t}_{g m}^{\prime}\right)
\end{gathered}
$$

Equations (1), (3) and (4) are written for a general streamline, while equation (2) is integrated from the inner radius $R_{i}$ to the outer radius $R_{e}$.

In (1) $H$ is the total enthalpy in its own reference frame, thus the absolute total enthalpy $\left(h_{t}\right)$ for stator rows or the relative total enthalpy $\left(h_{t r}\right)$ for rotor rows. Analogous considerations can be made for the other fundamental unknowns: the total pressure $P\left(p_{t}\right.$ or $\left.p_{t r}\right)$, the velocity magnitude $v(c$ or $w)$ and the flow angle $\varepsilon(\alpha$ or $\beta)$.

One can observe that, for the solution of the equation set from (1) to (4):

- there are further unknowns (called auxiliary unknowns) in addition to fundamental ones,

- all the fundamental unknowns are functions of the radius

The auxiliary unknowns are the density $\rho_{2}\left(r_{2}\right)$ in (2) and the fluid-dynamics parameters in (3) and (4). These quantities are functions of the fundamental unknowns and can be expressed by:

$$
\begin{gathered}
\rho_{2}=\frac{p_{t 2}}{R_{g} T_{t 2}}\left(\frac{p_{2}}{p_{t 2}}\right)^{\frac{1}{k}}=f\left(P_{2}, H_{2}, v_{2}, \varepsilon_{2}\right) \\
t_{f i}, t_{f l}^{\prime}=f\left(P_{2}, H_{2}, v_{2}, \varepsilon_{2}\right)
\end{gathered}
$$

To explicit the velocity magnitude $v_{2}(r)$ in (2) it is necessary to resort to the radial equilibrium equation [3] that, for the given assumptions, is:

$$
\frac{1}{\rho} \frac{d p}{d X}=\frac{v^{2} \operatorname{sen}^{2} \varepsilon}{R} \frac{d R}{d X}
$$

where $X$ is the direction of the motion equation projection line. As (7) is an integral equation, the constant of integration has to be evaluated. As the presented method is developed in terms of pressure, the value of the static pressure at the outlet section of the machine is given. Thus the classic constant of integration $v_{0}$ can be replaced with a first guess set of values for the static pressure in the control stations, which has to be updated during the iterative procedure till the value of the mass flow in each control station becomes consistent with the values of the other stations. 
To define how of total pressure $P(r)$ and flow angle $\varepsilon(r)$ depend on the radius, it is necessary to make use of local instead of overall correlative relationships. Furthermore, the total enthalpy at row exit $H(r)$ is known for the stator row case and it can be evaluated from the corresponding value of the absolute total enthalpy through the use of changing equations for the rotor row case. As the total pressure and the flow angle distributions can be evaluated only if the streamline radii are known, it is necessary to employ the continuity equation to determine these values:

$$
\int_{R_{i}}^{R} v_{2}(r) \rho_{2}(r) \cos \varepsilon_{2}(r) r d r=\dot{m}_{2}(R)
$$

For the real gas, one can employ thermodynamic tables which express the fluid properties as a function of two fundamental unknowns:

$$
\begin{gathered}
H_{2}=H_{1} \\
\varepsilon_{2}=f_{\varepsilon}\left(t^{\prime}{ }_{f l}, \bar{t}_{g m}^{\prime}\right)=f_{\varepsilon}\left(t^{\prime}{ }_{f l}\right)=f_{\varepsilon}\left(H_{2}, \varepsilon_{2}, P_{2}, v_{2}\right) \\
P_{2}=P_{1} f_{\sigma}\left(t^{\prime}{ }_{f i}, \bar{t}^{\prime}{ }_{g j}\right)=P_{1} f_{\sigma}\left(t^{\prime}{ }_{f i}\right)=P_{1} f_{\sigma}\left(H_{2}, \varepsilon_{2}, P_{2}, v_{2}\right)
\end{gathered}
$$

then the entropy is:

$$
s_{2}=f_{s}\left(H_{2}, P_{2}\right)
$$

thus the other thermodynamics quantities can be evaluated from the definition of total state:

$$
\begin{gathered}
h_{2}=f_{h}\left(s_{2}, \tilde{p}_{2}\right) \\
v_{2}=\sqrt{2\left(H_{2}-h_{2}\right)+u_{2}^{2}}
\end{gathered}
$$

\section{B. Resolution method}

As described in the previous paragraph, the resolution method proceeds through two different steps:

- In the first step the information of the imposed inlet total quantities is transferred to all control stations from upstream to downstream.

- In the second step the static pressure distribution is updated from the outlet section upstream in order to reduce the gap between the values of mass flow rate in two consecutive sections.

The calculation procedure runs till the continuity equation is satisfied in each control station.

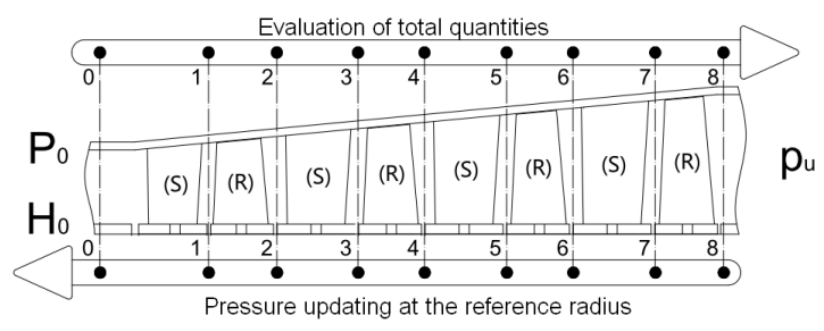

Fig. 2. Computational procedure diagram

Furthermore, it is necessary to employ a further iterative process in order to arrange the streamline position until the mass flow rate reaches the same value in each stream tube. This procedure is really important because during the calculation process the radial gradients of fundamental unknowns modify the velocity distribution, thus the continuity equation could not be satisfied for each stream tube. As first guess solution one can draw the streamline layout leaning on points whose radius subdivides the meridional channel into streamtubes with equal area.

\section{Calculation of fundamental unknowns at row exit}

Using the reference frame unknowns, the employed equations for the resolution procedure to evaluate the fundamental unknowns at row exit are the same for both stator and rotor rows [4]. Thus, if we indicate with 1 the inlet station and with 2 the outlet station of the general row, we can write, for sake of simplicity for a perfect gas, a general equations set:

$$
\begin{gathered}
H_{2}=H_{1} \\
P_{2}=P_{1} \sigma_{1,2}=P_{1}\left[1-\frac{\bar{\omega}}{1+\bar{\omega}}\left(1-\frac{\left(\tilde{p}_{2} / P_{1}\right)}{f_{u}^{\frac{k}{k-1}}}\right)\right] \\
\dot{m}_{2}=A_{2}^{*} \cos \varepsilon_{2} \frac{P_{1}}{\sqrt{H_{1}}} \sigma_{1,2}+ \\
-\frac{k}{k-1} \sqrt{2} \sqrt{\left[f_{u}\left(\frac{\tilde{p}_{2}}{P_{2}}\right)^{2 / k}-\left(\frac{\tilde{p}_{2}}{P_{2}}\right)^{k+1 / k}\right]} \\
\varepsilon_{2}\left(r_{2}\right)=f_{\varepsilon}\left(t_{f l}^{\prime}, \bar{t}_{g m}^{\prime}\right)
\end{gathered}
$$

where

$$
f_{u}=\left(1+\frac{u_{2}^{2}}{2 H_{1}}\right)
$$

The above equations set combined with the radial equilibrium equation is employed for each streamline. For rotor rows the radial equilibrium equation, written in terms of static pressure, in a form that we call simplified form due to the chosen simplifying assumptions, is:

$$
\frac{1}{\rho} \frac{d p}{d R}=\frac{w^{2} \operatorname{sen}^{2} \beta+u^{2}+2 u w \operatorname{sen} \beta}{R}
$$

One evaluates the density:

$$
\rho_{2}=\frac{p_{t r 2}}{R_{g} T_{t r 2}}\left(\frac{p_{2}}{p_{t r 2}}\right)^{\frac{1}{k}}
$$

thus the velocity magnitude:

$$
w_{2}^{2}=2\left(h_{t r 2}-h_{2}\right)=2 \frac{k}{k-1} R_{g} T_{t r 2}\left[1-\left(\frac{p_{2}}{p_{t r 2}}\right)^{\frac{k-1}{k}}\right]
$$

Then equations (19) and (20), combined with equation (18) lead to the expression:

$$
\begin{aligned}
& \left(\frac{d p}{d R}\right)_{2}=\frac{2 k}{k-1}\left[p_{t r 2} \frac{k-1}{k} p_{2}{ }^{\frac{1}{k}}-p_{2}\right] \frac{\operatorname{sen}^{2} \beta_{2}}{R}+ \\
& +2 \sqrt{\frac{2 k}{k-1}} \frac{p_{t r 2}}{\sqrt{R_{g} T_{t r 2}}} \sqrt{\left(\frac{p_{2}}{p_{t r 2}}\right)^{\frac{2}{k}}-\left(\frac{p_{2}}{p_{t r 2}}\right)^{\frac{k+1}{k}}} \operatorname{\omega sen} \beta_{2}+ \\
& +\frac{p_{t r 2}}{R_{g} T_{t r 2}}\left(\frac{p_{2}}{p_{t r 2}}\right)^{\frac{1}{k}} \omega^{2} R
\end{aligned}
$$


The whole equations set, matched with the radial equilibrium equation, is therefore combined with changing equations which express the relationship between the absolute and relative reference frame unknowns.

\section{First guess solution and pressure updating process}

Being the proposed procedure an outlet fixed pressure method, one has to employ as auxiliary unknown the static pressure in each control station. Thus one has to set a appropriate first guess value of static pressure distribution for each control station. In fact, even if the choice of the first guess solution can't modify the calculation results, it affects the iterative cycle convergence rate and above all its robustness and stability.

For the present work we chose as first guess solution a thermodynamic distribution of static pressure along the control stations, based on the evaluation of both isentropic enthalpy drop of the different stages and a fixed polytropic efficiency of the machine.

To better understand the static pressure updating process it is useful to consider a single stage of the turbine and indicate with 1 the inlet station, with 2 the intermediate station between stator and rotor and finally with 3 the outlet station. As the inlet total parameters and the outlet static pressure are given, a variation of static pressure $p_{2}$ leads to opposite variations in mass flow rate values through stator $\left(\dot{m}_{2}\right)$ and rotor $\left(\dot{m}_{3}\right)$. Thus, a simple $\mathrm{p}_{2}$ updating process can be employed as updating weight to the difference between $\dot{m}_{2}$ and $\dot{m}_{3}$, that must be clearly equal to zero at convergence.

\section{Computer code}

A Fortran language computer code, based on equations illustrated in the previous paragraph, has been developed. In Fig. 4 its block diagram, with main convergence processes, is presented. The integration process of radial equilibrium equation has been developed in two different ways: the Euler method and a four step Runge-Kutta model. For the present application these procedures provide very closed outputs, thus the integration method doesn't affect the results significantly. For this reason the more simple Euler method has been employed. The code has been applied to both an experimental multistage gas turbine investigated at the University of Hannover [5] and a small multistage industrial steam turbine in order to assess the method skills to predict the fundamental unknowns of different kind of axial turbine (gas and steam turbines) which are employed in combined cycle plants. The experimental turbine has been widely investigated both experimentally and with numerical calculations [6]. Thus, several experimental and calculation's results are available in open literature.

Despite, in order to provide complete value of unknowns for all machine sections, a CFD 3D viscous calculation with software NUMECA [7], has been employed.

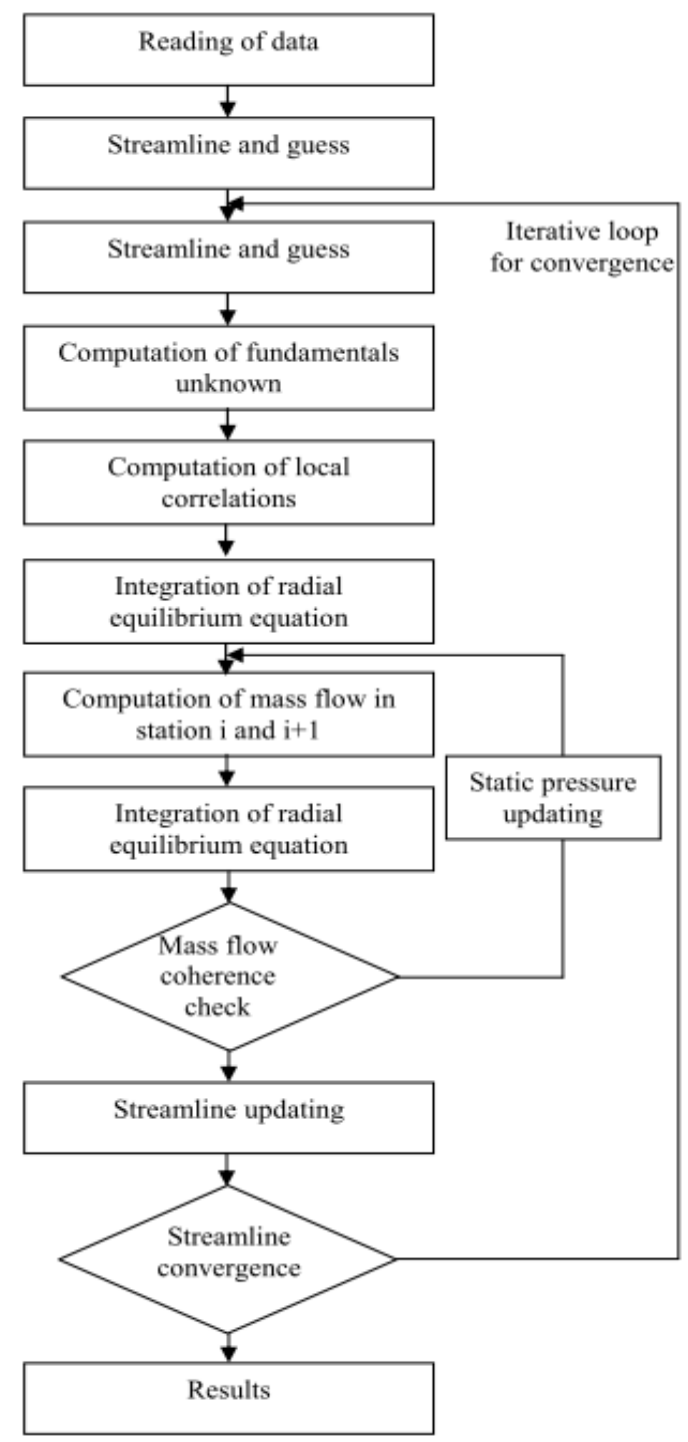

Fig. 4. Computer code block diagram

\section{A. Application with imposed correlative parameters}

In a first application correlative parameters are assigned (flow angle and total to total loss coefficient) in order to evaluate the mismatch between the procedure and the full 3D calculation results. These differences are due to only given assumptions and don't consider local correlation procedures evaluation error. Thus, one imposes the circumferentially averaged correlative parameters from the results of the 3D CFD calculation:

$$
\begin{gathered}
\varepsilon_{2}(r)=\bar{\varepsilon}_{2}(r) \\
\sigma_{1,2}(r)=\bar{\sigma}_{1,2}(r)
\end{gathered}
$$

In Fig. 5 the results from the proposed method and from the $3 \mathrm{D}$ CFD procedure for the experimental gas turbine, are compared. The code has demonstrated high stability, quick convergence rate, and the results are in good agreement with those from the CFD calculation. 

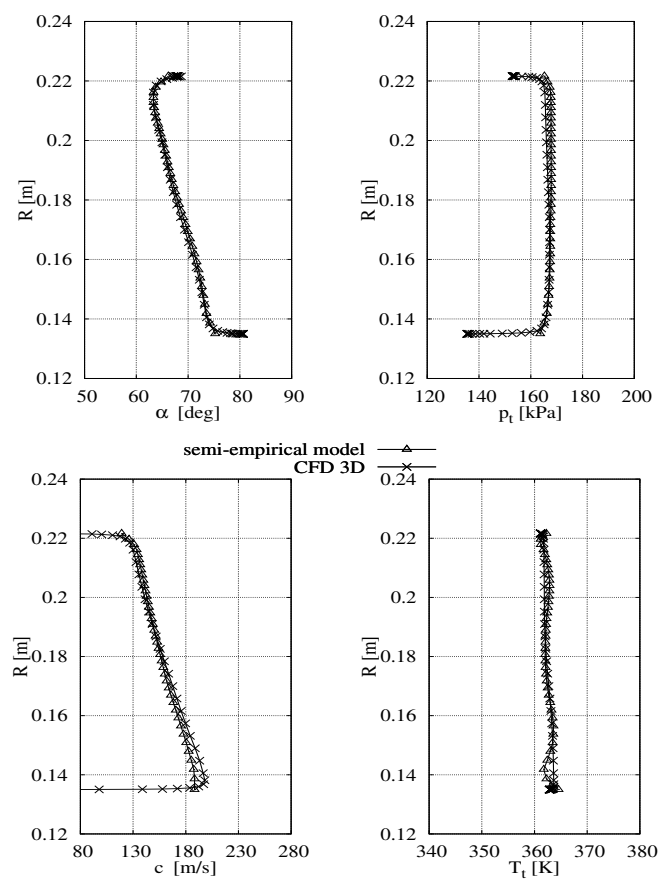

Fig. 5. Comparison of fundamental unknowns in the $6^{\text {th }}$ control station $\left(3^{\text {rd }}\right.$ stator row exit) for the experimental turbine

In Fig. 6 and 7 the comparison between the results of the proposed method and those of the CFD 3D procedure and an industrial method for the industrial steam turbine, is presented.
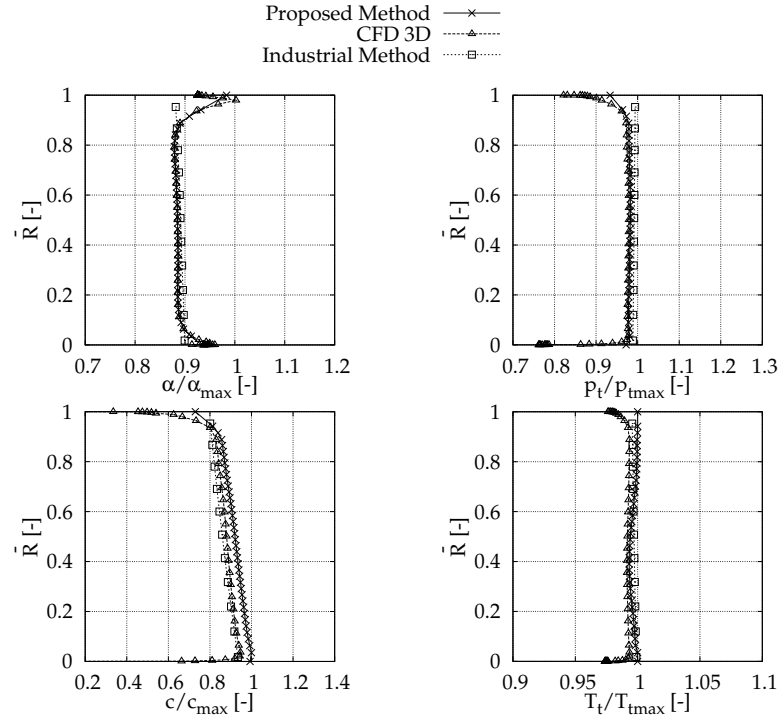

Fig. 6. Comparison of fundamental unknowns in the $6^{\text {th }}$ control station $\left(3^{\text {rd }}\right.$ stator row exit) for the industrial steam turbine

The results are in a normalized form for confidentiality. Also for this application, the code, which employs appropriate thermodynamic steam tables, translated in Fortran language with appropriate subroutines, has provided reliable radial distributions of fundamental unknowns in accordance with both the 3D CFD calculation and the industrial method results, confirming its skills of robustness and high convergence rate.

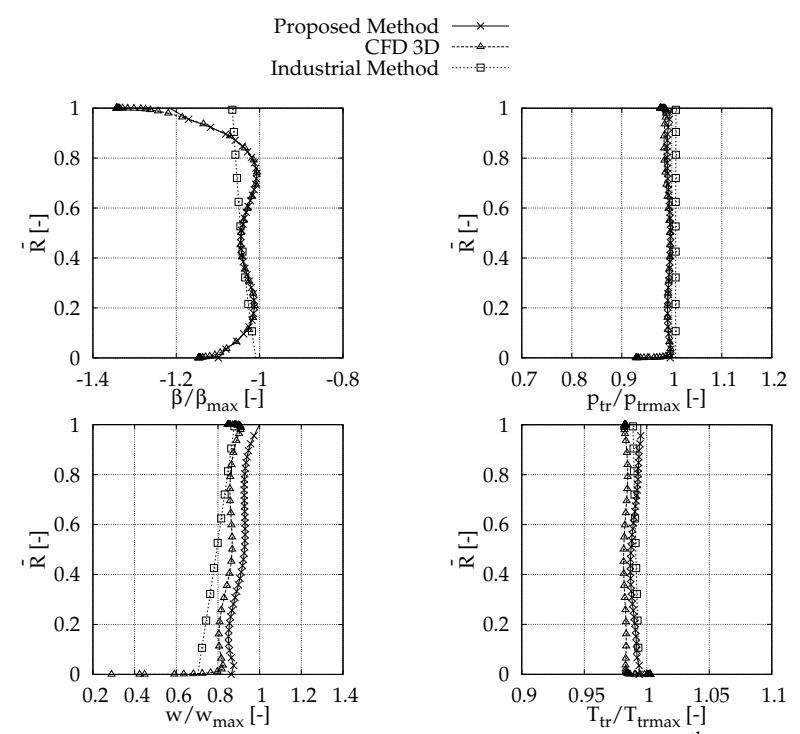

Fig. 7. Comparison of fundamental unknowns in the $6^{\text {th }}$ control station $\left(3^{\text {rd }}\right.$ rotor row exit) for the industrial steam turbine

\section{B. Application with local correlative equations}

In a further application the assignment of correlative parameters has been replaced with the introduction of appropriate local correlative equations able to provide the radial distribution of both loss coefficient and flow angle. For the evaluation of loss coefficient one chooses the model developed by Traupel [8], combined with the Petrovic distribution's law [9]. For the evaluation of flow deviation angle a correlative equation model, proposed in a previous work by the author [10], has been employed. In Fig. 8 the comparison between the results of the proposed method with local correlative equations and those of the CFD 3D procedure for the experimental steam turbine for example at 3rd stator row exit is presented. Also in this application the code provides reliable radial distribution for the four fundamental unknowns which are consistent with the circumferentially averaged results of the 3D CFD calculation.
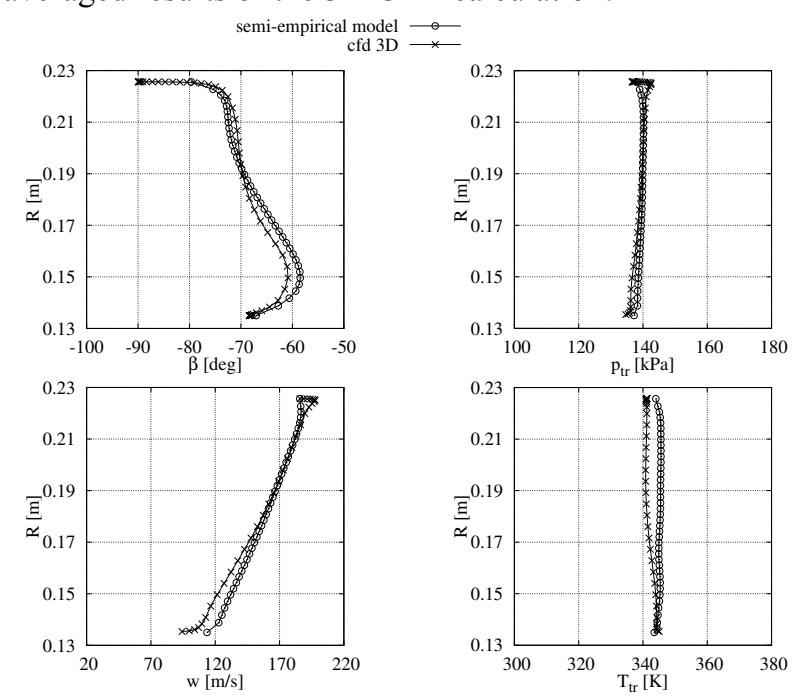

Fig. 7. Comparison of fundamental unknowns in the $7^{\text {th }}$ control station $\left(3^{\text {rd }}\right.$ rotor row exit) for the experimental turbine 


\section{Conclusion}

A two-dimensional meridional computational method based on the use of the radial equilibrium equation for the analysis of multi stage axial turbines has been proposed. A computer code, written in Fortran language and based on the proposed method, has been developed. Thus the code has been employed to investigate both multi-stage axial gas turbine test case described in the open literature and a multistage industrial steam turbine. In a first application, in order to evaluate the stability and robustness of the procedure, the code has been applied by assigning the correlative parameters. The results have been compared with results of complex 3D procedures; they show high stability, quick convergence rate and above all reliable distributions of fundamental unknowns along the radial direction for both kind of turbine. In a second application the code, combined with correlative equations providing radial distributions of both flow angle and total to total loss coefficient, has been employed. The obtained results confirm the procedure skills and show good agreement with circumferentially averaged results from the 3D CFD method. The achieved results confirm that the proposed method can be employed for a simplified analysis of radial distributions of fundamental unknowns which provide the machine characteristics. The procedure versatility, demonstrated in the applications of the code to both a gas and a steam turbine, confirms that the method can be employed as a valid instrument to evaluate the overall performance of the turbine in order to ascertain if it is possible to employ the investigated machine in a combined cycle.

\section{Acknowledgement}

This work has been developed in the ECOMOS-FIRB Project.

\section{References}

[1] AA. VV., Throughflow Calculations in Axial Turbomachinery, AGARD CP-195, 1976.

[2] L. Ratto, A. Satta, A simplified method with local correlations for the analisys of multi stage axial turbines, Proceedings of ASME Turbo Expo, Glasgow, 2010.

[3] A. Bosio, G. Croce, M. Marini, A. Satta, L'equazione del gradiente della velocità per il calcolo through flow nelle turbomacchine, Convegno Turbomacchine, 1996.

[4] G. Croce, E. Dedicato, R. Gazzano, L. Ratto, A. Satta, Procedure for the axial multi stage turbomachineries performance analisys, ISROMAC-12, Honolulu (USA), 2008.

[5] L. Fottner (Ed.), Test cases for computation of internal flows in aero engine components, AGARD Advisory Report 275, Advisory Group for Aeronautical Research and Development, Paris, 1990, pp. 365-375.

[6] G. Gerolymos, C. Hanish, Multistage three-dimensional Navier-Stokes computation of off-design operation of a fourstage turbine, Proceedings of the Institution of Mechanical Engineers, Part A: Journal of Power and Energy, vol. 213, pp. 243-261, 1999.

[7] NUMECA, User's manual, educational license, 2010.

[8] W. Traupel, Thermische Turmomaschinen, vol. 13 rd Ed., Sprinter, Berlin-Heidelberg-New York, 1977.

[9] M. Petrovic, W. Riess, Off-design flow analysis and performance prediction of axial turbines, Proceedings of ASME 97-GT-55, 1997.
[10] A. Massardo, A.Satta, A Correlation for the Secondary Deviation Angle, The 1985 Beijing International Gas Turbine Symposium and Exposition, Beijing, 1985.

\section{Nomenclature}

$$
\begin{aligned}
& A^{*} \quad \text { passage section orthogonal to the velocity }\left[\mathrm{m}^{2}\right] \\
& f_{\varepsilon} \quad \text { angle correlation } \\
& f_{\sigma} \quad \text { loss correlation } \\
& f_{u} \quad\left(1+\frac{u_{2}^{2}}{2 H_{1}}\right) \quad \text { [-] } \\
& h \quad \text { thermodynamic enthalpy }[\mathrm{J} / \mathrm{kg}] \\
& H \text { total enthalpy }\left(h_{t}\right) \text { or relative total enthalpy }\left(h_{t r}\right) \\
& \text { H }[\mathrm{J} / \mathrm{kg}] \\
& k \quad\left\lfloor C_{p} / C_{v}\right\rfloor \text { heat capacity ratio [-] } \\
& \dot{m} \quad \text { mass flow rate }[\mathrm{kg} / \mathrm{s}] \\
& \text { M Mach number [-] } \\
& p \quad \text { thermodynamic pressure }[\mathrm{Pa}] \\
& P \quad \text { total } \\
& R_{g} \quad \text { specific gas constant }[\mathrm{J} / \mathrm{kgK}] \\
& \bar{R} \quad \text { normalized radius [-] } \\
& s \quad \text { entropy }[\mathrm{J} / \mathrm{kgK}] \\
& t_{f i}, t_{f l}^{\prime} \quad \text { fluid-dynamics parameters for local correlations } \\
& t_{g j}, t_{g m}^{\prime} \text { geometrical parameters for local correlations } \\
& T \quad \text { temperature }[K] \\
& u \quad \text { blade } \operatorname{speed}[\mathrm{m} / \mathrm{s}] \\
& v \quad \text { absolute }(c) \text { or relative }(w) \text { speed }[\mathrm{m} / \mathrm{s}] \\
& X \quad \text { motion equation projection line }
\end{aligned}
$$

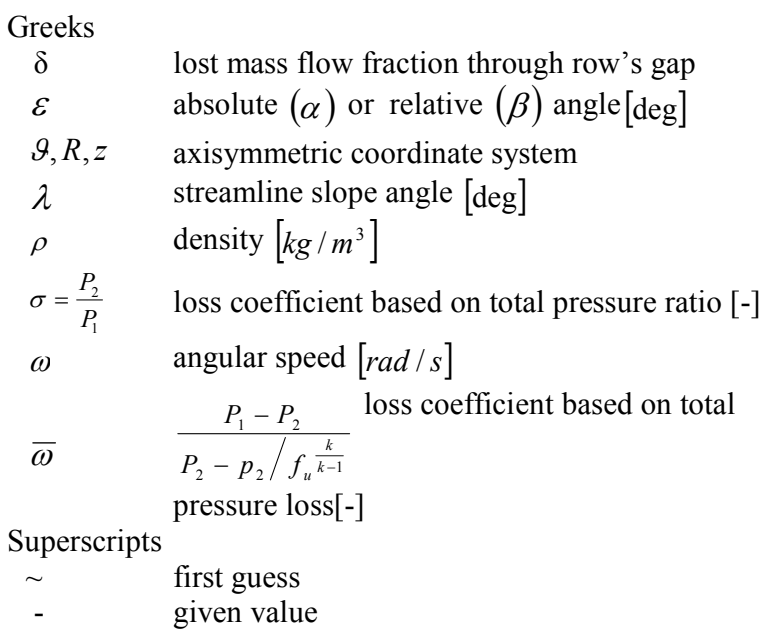

$\begin{array}{ll}\text { Subscripts } & \\ 0 & \text { referral status } \\ 1,2,3 & \text { control stations } \\ f & \text { lost, referred to the mass flow } \\ e & \text { outer } \\ i & \text { inner } \\ s & \text { isentropic } \\ t & \text { total } \\ t r & \text { total relative } \\ u & \text { referred to the blade speed }\end{array}$

